Preprints of the

Max Planck Institute for

Research on Collective Goods

Bonn 2008/47

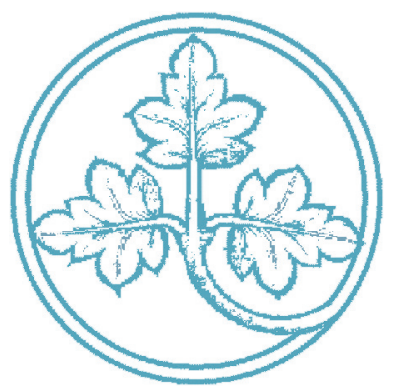

A Generalization of the Atkinson-Stiglitz (1976) Theorem on the Undesirability of Nonuniform Excise Taxation

Martin Hellwig

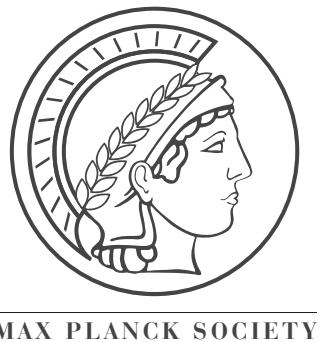




\title{
A Generalization of the Atkinson-Stiglitz (1976) Theorem on the Undesirability of Nonuniform Excise Taxation
}

\author{
Martin Hellwig
}

December 2008 


\title{
A Generalization of the Atkinson-Stiglitz (1976) Theorem on the Undesirability of Nonuniform Excise Taxation*
}

\author{
Martin F. Hellwig \\ Max Planck Institute for Research on Collective Goods \\ Kurt-Schumacher-Str. 10, D-53113 Bonn, Germany \\ hellwig@coll.mpg.de
}

December 3, 2008

\begin{abstract}
The Atkinson-Stiglitz (1976) theorem on the undesirability of nonuniform excise taxation when all agents have homogeneous, separable preferences is extended to allow for nonseparability with respect to endogenous variables that will be subject to distortions. The result is useful for analysing models with production and education. For such models, the conclusion of Bovenberg and Jacobs (2005), that it is desirable to distort production rather than education choices, is shown to be reversed if the hidden characteristic affects the cost of education rather than productivity.

Key Words: Optimal Taxation, Atkinson-Stiglitz Theorem.

JEL Classification: H21.
\end{abstract}

\section{Introduction}

A well known result by Atkinson and Stiglitz (1976) shows that, under certain homogeneity and separability assumptions on preferences, an optimal system of taxes for public-sector funding or for redistribution relies on direct taxation only. Kaplow (2006) and Laroque (2005) have recently extended this result to show that, under the assumptions of Atkinson and Stiglitz,

${ }^{*}$ I am grateful for discussions with Guy Laroque and for helpful comments from Felix Bierbrauer, Christoph Engel, and Andreas Nicklisch. 
any feasible, incentive-compatible allocation is Pareto-dominated by an allocation that can be implemented by direct taxation, without recourse to distortionary indirect taxes. This note uses the argument of Kaplow (2006) and Laroque (2005) to show that their result is a special case of a more general theorem with a weaker separability assumption on preferences. ${ }^{1}$ It also gives an example with production and education where this weakening of separability is relevant, showing that the results of Bovenberg and Jacobs (2005) are sensitive to the way the hidden characteristics enter the model.

\section{The Setup}

I consider a model in which agents care about outcomes that are specified as $n$-dimensional vectors $\left(x_{1}, \ldots, x_{n}\right) \in \mathbb{R}^{n}$. An agent's preferences over outcomes depend on his "type", a parameter $t$ that lies in an interval $\left[t_{0}, t_{1}\right]$. His preferences are represented by a utility function $u$, which is assumed to take the separable form

$$
u\left(x_{1}, \ldots, x_{n}, t\right)=v\left(\varphi\left(x_{1}, \ldots, x_{n}\right), x_{n}, t\right),
$$

where $v$ and $\varphi$ are continuously differentiable functions, with derivatives satisfying $v_{\varphi}>0$ and $\varphi_{i}>0$ for all $i$. The function $v$ is also assumed to satisfy the single-crossing condition that $\frac{v_{x_{n}}}{v_{\varphi}}$, the marginal rate of substitution between $\varphi$ and $x_{n}$, is everywhere increasing in $t$.

In Atkinson and Stiglitz (1976), Kaplow (2006), and Laroque (2005), the outcome variables $x_{1}, \ldots, x_{n-1} \geq 0$ would be consumption levels of different goods, $x_{n}=-y$, where $y$ is the level of output that the agent produces, and $t$ a productivity parameter, with the interpretation that $\ell=\frac{y}{t}$ is the amount of labour the agent needs to put in to produce the output $y$. In their work, $u$ takes the form

$$
u\left(x_{1}, \ldots, x_{n-1}, \frac{-x_{n}}{t}\right)=v\left(\varphi\left(x_{1}, \ldots, x_{n-1}\right), \frac{-x_{n}}{t}\right),
$$

which clearly is a special case of (1). Besides allowing for a more general form of the dependence of utility on $t,(1)$ differs from (2) by allowing for the possibility that the subutility $\varphi$ may depend on all outcome variables, rather than just $x_{1}, \ldots, x_{n-1}$. This difference plays a role in a model of education and production with the specification

$$
u=\varphi\left(c, \frac{y}{z}\right)-e(z, t),
$$

\footnotetext{
${ }^{1}$ A similar generalization is obtained by Gauthier and Laroque (2008).
} 
where $x_{1}=c$ is the agent's consumption, $x_{2}=-y$ is the agent's output, and $x_{3}=z$ is the agent's labour productivity. The labour productivity is identified with the education level $z$. To reach this level, the agent must spend the effort $e(z, t)$, which depends on his type $t$ as well as the education level $z$. In this specification, by contrast to (2), the agent's type $t$ affects the cost of reaching the productivity level (or education level) $z$, rather than the productivity level itself.

\section{Incentive Compatibility}

An agent's type is assumed to be his private information. An allocation is a mapping $t \rightarrow\left(x_{1}(t), \ldots, x_{n}(t)\right)$ that indicates how outcomes depend on types. An allocation is incentive-compatible on the interval $\left[t_{0}, t_{1}\right]$ if

$$
u\left(x_{1}(t), \ldots, x_{n}(t), t\right) \geq u\left(x_{1}\left(t^{\prime}\right), \ldots, x_{n}\left(t^{\prime}\right), t\right)
$$

for all $t$ and $t^{\prime}$ in $\left[t_{0}, t_{1}\right]$. Under the separability assumption (1), this condition is equivalent to the requirement that

$$
v\left(w(t), x_{n}(t), t\right) \geq v\left(w\left(t^{\prime}\right), x_{n}\left(t^{\prime}\right), t\right),
$$

for all $t$ and $t^{\prime}$ in $\left[t_{0}, t_{1}\right]$, where, for each $t$,

$$
w(t):=\varphi\left(x_{1}(t), \ldots, x_{n}(t)\right) .
$$

Incentive compatibility depends only on $x_{n}(t)$ and on the subutility $w(t)$ that is given by (6). Which combination of the outcome variables $x_{1}, \ldots, x_{n-1}$ is combined with $x_{n}(t)$ to achieve the subutility $w(t)$ is irrelevant for incentive compatibility. Formally, one obtains:

Lemma 1 Let $\left(x_{1}(\cdot), \ldots, x_{n}(\cdot)\right)$ be an incentive-compatible allocation. For each $t$, let $w(t)$ be given by (6), and let $\hat{x}_{1}(t), \ldots, \hat{x}_{n-1}(t)$ be such that

$$
\varphi\left(\hat{x}_{1}(t), \ldots, \hat{x}_{n-1}(t), x_{n}(t)\right)=w(t) .
$$

Then the allocation $\left(\hat{x}_{1}(\cdot), \ldots, \hat{x}_{n-1}(\cdot), x_{n}(\cdot)\right)$ is also incentive-compatible.

The observation that incentive compatibility reduces to condition (5) brings the analysis into the domain of known results about incentive compatibility. Given that $v$ satisfies a single-crossing conditions, by well-known arguments yield: ${ }^{2}$

\footnotetext{
${ }^{2}$ The first version of this lemma is to be found in Mirrlees (1976). A proof without differentiability assumptions on the function $V(\cdot)$ is given in Hellwig (2006/2008).
} 
Lemma 2 An allocation $\left(x_{1}(\cdot), \ldots, x_{n}(\cdot)\right)$ is incentive-compatible on $\left[t_{0}, t_{1}\right]$ if and only if $x_{n}(\cdot)$ is a nondecreasing function and, for all $t$ and $t^{\prime}$ in $\left[t_{0}, t_{1}\right]$, one has

$$
V(t)-V\left(t^{\prime}\right)=\int_{t^{\prime}}^{t} v_{t}\left(w(\tau), x_{n}(\tau), \tau\right) d \tau
$$

where

$$
V(t):=v\left(w(t), x_{n}(t), t\right)
$$

\section{Feasibility and Dominance}

Let $F$ be the cross-section distribution of agents' types, where $F$ has support $\left[t_{0}, t_{1}\right]$. An allocation is said to be feasible if the aggregates $\int x_{i}(t) d F(t)$, $i=1, \ldots, n$, satisfy the resource constraint

$$
\sum_{i=1}^{n} p_{i} \int x_{i}(t) d F(t) \leq K
$$

where $p_{1}, \ldots, p_{n} \geq 0$ and $K$ are given constants. If the inequality in (10) is strict, the allocation is said to be strictly feasible.

Lemma 3 Let $\left(x_{1}(\cdot), \ldots, x_{n}(\cdot)\right)$ be feasible and incentive-compatible on $\left[t_{0}, t_{1}\right]$ and assume that

$$
\sum_{i=1}^{n-1} p_{i} x_{i}(t)>\min _{x_{1}, \ldots, x_{n-1}} \sum_{i=1}^{n-1} p_{i} x_{i} \text { s.t. } \varphi\left(x_{1}, \ldots, x_{n-1}, x_{n}(t)\right) \geq w(t)
$$

for a nonnegligible set of types, where $w(t)$ is given by (6). Then there exists an allocation $\left(\hat{x}_{1}(\cdot), \ldots, \hat{x}_{n-1}(\cdot), \hat{x}_{n}(\cdot)\right)$ with $\hat{x}_{n}(\cdot)=x_{n}(\cdot)$ that is strictly feasible and incentive-compatible on $\left[t_{0}, t_{1}\right]$ and that generates the same payoffs as the allocation $\left(x_{1}(\cdot), \ldots, x_{n}(\cdot)\right)$.

Proof. For each $t$, if $x_{1}(t), \ldots, x_{n-1}(t)$ minimizes $\sum_{i=1}^{n-1} p_{i} x_{i}$ under the constraint that $\varphi\left(x_{1}, \ldots, x_{n-1}, x_{n}(t)\right) \geq w(t)$, let $\hat{x}_{i}(t)=x_{i}(t), i=1, \ldots, n-1$; if (11) holds, let $\hat{x}_{1}(t), \ldots, \hat{x}_{n-1}(t)$ be such that

$$
\sum_{i=1}^{n-1} p_{i} \hat{x}_{i}(t)<\sum_{i=1}^{n-1} p_{i} x_{i}(t)
$$


and, moreover, $\varphi\left(\hat{x}_{1}(t), \ldots, \hat{x}_{n-1}(t), x_{n}(t)\right)=w(t)$. By construction, the allocation $\left(\hat{x}_{1}(\cdot), \ldots, \hat{x}_{n-1}(\cdot), x_{n}(\cdot)\right)$ is strictly feasible and payoff-equivalent to the allocation $\left(x_{1}(\cdot), \ldots, x_{n}(\cdot)\right)$. By Lemma 1 , it is also incentive-compatible on $\left[t_{0}, t_{1}\right]$.

If the surplus of the allocation $\left(\hat{x}_{1}(\cdot), \ldots, \hat{x}_{n-1}(\cdot), x_{n}(\cdot)\right)$ can be redistributed to the participants without upsetting incentive compatibility, the allocation $\left(x_{1}(\cdot), \ldots, x_{n}(\cdot)\right)$ in Lemma 3 is actually Pareto-dominated. This is the point of:

Lemma 4 If $\left(x_{1}(\cdot), \ldots, x_{n}(\cdot)\right)$ satisfy the assumptions of Lemma 3. there exists an $\left(\bar{x}_{1}(\cdot), \ldots, \bar{x}_{n}(\cdot)\right)$ that is feasible and incentive-compatible on $\left[t_{0}, t_{1}\right]$ and that provides every type with a payoff that is strictly greater than his payoff under the allocation $\left(x_{1}(\cdot), \ldots, x_{n}(\cdot)\right)$.

Proof Sketch. Given the allocation $\left(x_{1}(\cdot), \ldots, x_{n}(\cdot)\right)$, let $V(\cdot)$ and $w(\cdot)$ be given by (9) and (6) and, for any $\Delta \geq 0$, consider the integral equation

$$
W(t, \Delta)=W\left(t_{0}, \Delta\right)+\int_{t_{0}}^{t} v_{t}\left(\bar{w}(\tau, \Delta), x_{n}(\tau), \tau\right) d \tau,
$$

with the initial condition

$$
W\left(t_{0}, \Delta\right)=V\left(t_{0}\right)+\Delta
$$

where, for any $\tau$,

$$
\bar{w}(\tau, \Delta):=v^{-1}\left(W(\tau, \Delta), x_{n}(\tau), \tau\right)
$$

is the value of the subutility $\varphi$ that is needed if type $\tau$ is to have the payoff $W(\tau, \Delta)$ when the $n$-th outcome is given as $x_{n}(\tau)$. It is straightforward to show that, for any $\Delta \geq 0$, the function $W(\cdot, \Delta)$ is well defined, and that $W(t, \Delta)$ is increasing in $\Delta$, as well as jointly continuous in $t$ and $\Delta ;^{3}$ moreover, one has $W(t, 0)=V(t)$ and $\bar{w}(t, 0)=w(t)$ for all $t$.

Next, let $\left(\hat{x}_{1}(\cdot), \ldots, \hat{x}_{n-1}(\cdot), \hat{x}_{n}(\cdot)\right)$ be the allocation that is given by Lemma 3 . For any $\Delta$, let $\left(\bar{x}_{1}^{\Delta}(\cdot), \ldots, \bar{x}_{n}^{\Delta}(\cdot)\right)$ be such that $\bar{x}_{n}^{\Delta}(\cdot)=x_{n}(\cdot), \bar{x}_{i}^{\Delta}(\cdot)=\hat{x}_{i}(\cdot)$ for $i=2, \ldots, n-1$, and by fixing $\bar{x}_{1}^{\Delta}(\cdot)$ so that, for any $t$,

$$
\varphi\left(\bar{x}_{1}^{\Delta}(\cdot), \hat{x}_{2}(\cdot), \ldots, \hat{x}_{n-1}(\cdot), x_{n}(\cdot)\right)=\bar{w}(\cdot, \Delta) .
$$

By construction, for any $t \in\left[t_{0}, t_{1}\right]$, the allocation $\left(\bar{x}_{1}^{\Delta}(\cdot), \ldots, \bar{x}_{n}^{\Delta}(\cdot)\right)$ generates the utility level $W(t, \Delta)$. Because $W(t, \Delta)$ is increasing in $\Delta$, for

\footnotetext{
${ }^{3}$ For details of the argument, see the Appendix to Hellwig (2008 a).
} 
$\Delta>0$, one has $W(t, \Delta)>V(t)$. If $\Delta$ is sufficiently small, the allocation $\left(\bar{x}_{1}^{\Delta}(\cdot), \ldots, \bar{x}_{n}^{\Delta}(\cdot)\right)$ is uniformly close to the strictly feasible allocation $\left(\hat{x}_{1}(\cdot), \ldots, \hat{x}_{n-1}(\cdot), x_{n}(\cdot)\right)$ and must itself be feasible. By Lemma 2 , it is also incentive-compatible on $\left[t_{0}, t_{1}\right]$.

By the taxation principle of Hammond (1979) and Guesnerie (1995), one now obtains the following extension of the Atkinson-Stiglitz theorem.

Theorem 5 If the allocation $\left(x_{1}(\cdot), \ldots, x_{n}(\cdot)\right)$ is Pareto-optimal in the set of feasible, incentive-compatible allocations, there exists a tax schedule $T(\cdot)$, defined on the range of the function $x_{n}(\cdot)$, such that, for almost all $t \in$ $\left[t_{0}, t_{1}\right],\left(x_{1}(t), \ldots, x_{n}(t)\right)$ maximizes $u\left(x_{1}, \ldots, x_{n}, t\right)$ under the constraints that $x_{n}$ belong to the range of the function $x_{n}(\cdot)$ and that

$$
\sum_{i=1}^{n} p_{i} x_{i}+T\left(x_{n}\right) \leq 0 .
$$

Proof. For any $x_{n}$ and $w$, let $\hat{x}_{1}\left(x_{n}, w\right), \ldots, \hat{x}_{n-1}\left(x_{n}, w\right)$ be a solution to the problem of minimizing $\sum_{i=1}^{n-1} p_{i} x_{i}$ under the constraint that $\varphi\left(x_{1}, \ldots, x_{n}\right) \geq$ $w$, and let $E\left(x_{n}, w\right)=\sum_{i=1}^{n-1} p_{i} \hat{x}_{i}\left(x_{n}, w\right)$. By Lemmas 3 and 4 and the Paretooptimality of the allocation $\left(x_{1}(\cdot), \ldots, x_{n}(\cdot)\right)$, one must have

$$
\sum_{i=1}^{n-1} p_{i} x_{i}(t)=E\left(x_{n}(t), w(t)\right)
$$

for almost all $t \in\left[t_{0}, t_{1}\right]$, where, again $w(t)$ is given by (6).

For any $t \in\left[t_{0}, t_{1}\right]$, let

$$
\tau(t)=-E\left(x_{n}(t), w(t)\right)-p_{n} x_{n}(t) .
$$

I claim that, for any $t$ and $t^{\prime}, x_{n}(t)=x_{n}\left(t^{\prime}\right)$ implies $\tau(t)=\tau\left(t^{\prime}\right)$. For suppose that $x_{n}(t)=x_{n}\left(t^{\prime}\right)$ and $\tau(t) \neq \tau\left(t^{\prime}\right)$. If $\tau(t)>\tau\left(t^{\prime}\right)$, then, by (17), one must have $w(t)<w\left(t^{\prime}\right)$. By the monotonicity of $v$, it follows that $v\left(w(t), x_{n}(t)\right)<v\left(w\left(t^{\prime}\right), x_{n}(t)\right)=v\left(w\left(t^{\prime}\right), x_{n}\left(t^{\prime}\right)\right)$, contrary to the assumption that the allocation $\left(x_{1}(\cdot), \ldots, x_{n}(\cdot)\right)$ is incentive-compatible. The assumption that $x_{n}(t)=x_{n}\left(t^{\prime}\right)$ and $\tau(t)>\tau\left(t^{\prime}\right)$ thus leads to a contradiction and must be false. A precisely symmetric argument eliminates the possibility that $x_{n}(t)=x_{n}\left(t^{\prime}\right)$ and $\tau(t)<\tau\left(t^{\prime}\right)$.

Because $x_{n}(t)=x_{n}\left(t^{\prime}\right)$ implies $\tau(t)=\tau\left(t^{\prime}\right)$, there exists a function $T(\cdot)$, defined on the range of $x_{n}(\cdot)$ such that, for any $t \in\left[t_{0}, t_{1}\right]$, one has $\tau(t)=$ 
$T\left(x_{n}(t)\right)$. Thus, if $x_{n}=x_{n}\left(t^{\prime}\right)$ for some $t^{\prime} \in\left[t_{0}, t_{1}\right]$ and $\left(x_{1}, \ldots, x_{n}\right)$ satisfies (15), one has

$$
\sum_{i=1}^{n-1} p_{i} x_{i} \leq E\left(x_{n}\left(t^{\prime}\right), w\left(t^{\prime}\right)\right)
$$

and, hence,

$$
u\left(x_{1}, \ldots, x_{n}, t\right) \leq v\left(w\left(t^{\prime}\right), x_{n}\left(t^{\prime}\right), t\right),
$$

regardless of $t$. By incentive-compatibility, it follows that

$$
u\left(x_{1}, \ldots, x_{n}, t\right) \leq v\left(w(t), x_{n}(t), t\right)=u\left(x_{1}(t), \ldots, x_{n}(t), t\right)
$$

for any such $\left(x_{1}, \ldots, x_{n}\right)$ and any $t$.

Remark 6 The tax schedule $T(\cdot)$ in Theorem 5 can be extended to any compact interval containing the range of the function $x_{n}(\cdot)$. For any $x_{n}$ that does not belong to this range, one just has to choose the tax $T\left(x_{n}\right)$ to be large enough so that no type would like to choose a vector $\left(x_{1}, \ldots, x_{n}\right)$ which gives rise to this high tax.

Remark 7 The feasibility condition (10) can be replaced by the more general condition $G\left(\int x_{1}(t) d F(t), \ldots, \int x_{n}(t) d F(t)\right) \leq 0$ if convexity assumptions on preferences and technology make it possible to work with a vector $\left(p_{1}, \ldots, p_{n-1}\right)$ of producer prices as in the second welfare theorem.

Remark 8 The extension of the Atkinson-Stiglitz theorem that is provided by Theorem 5 is not available for Deaton's (1979) theorem on the undesirability of nonuniform excise taxation when income taxes are affine and preferences over consumption goods are separable from labour-leisure choices, homothetic, and identical across agents. Although the basic argument of Kaplow (2006) and Laroque (2005) can also be used to prove Deaton's theorem, ${ }^{4}$ in the more general case, when $x_{n}$ is an argument of $\varphi$, there is no reason to believe that the tax schedule which is obtained from the taxation principle is going to be affine.

\section{Discussion}

Theorem 5 shows that, under the given separability and single-crossing conditions, any Pareto-optimal incentive-compatible allocation can be implemented by means of a tax system that leaves the relative prices of $x_{1}, \ldots, x_{n-1}$

\footnotetext{
${ }^{4}$ This is shown in Hellwig (2008 b).
} 
unchanged. Under the given differentiability assumptions on $\varphi$, it follos that any tax system which distorts the relative prices of $x_{1}, \ldots, x_{n-1}$ is inherently unsuitable for achieving Pareto optimality in the set of feasible, incentivecompatible allocations.

If we think of $x_{1}, \ldots, x_{n-1}$ as consumption goods and of $x_{n}$ as the amount of leisure that is left after producing, say, the output $y=1-x_{n}$ of an intermediate good, this corresponds to the Kaplow-Laroque version of the Atkinson-Stiglitz result that nonuniform indirect taxation is undesirable. However, the theorem applies more generally. For example, in the model of education and production with utility specification $u=\varphi\left(c, \frac{y}{z}\right)-e(z, t)$ that was sketched in the introduction, Theorem 5 implies that one should not have a distortionary tax on income, only a distortionary tax on education! ${ }^{5}$

This finding is at odds with Bovenberg and Jacobs (2005), who call for a distortionary tax on income and, possibly, subsidies to education. The difference is due to their working with the utility specification (in my notation) $u=c-\gamma\left(\frac{y}{t z}\right)-e(z)$, for which Theorem 5 indeed calls for distortionary taxation of income, with no distortions in education choices. In their specification, the hidden characteristic affects a person's cost of producing the output $y$, in my specification, it affects a person's cost of attaining the education level $z$.

It is bothersome to see that such substantive conclusions should depend on such a fine point as whether a person's "type" affects the person's productivity or the person's cost of attaining a given education level. In general, an agent's "type" should be a multi-dimensional characteristic which affects both. More precisely, it makes sense to think of both the productivity of an agent with education level $z$ and the cost of achieving this education level as being unobservable, determined by one or several hidden characteristics. The question then should be how these two information problems interact and how this interaction affects optimal utilitarian taxation. Recognizing this as an issue provides a good basis for further research on the respective roles of heterogeneities in productivities and in education costs.

\section{References}

[1] Atkinson, A.B., and J.E. Stiglitz (1976), The Design of Tax Structure: Direct versus Indirect Taxation, Journal of Public Economics 6, 55 75.

\footnotetext{
${ }^{5}$ I first became aware of Theorem 5 when I computed optimal tax formulae for this model and was surprised to see income taxation dropping out!
} 
[2] Bovenberg, A.L., and B. Jacobs (2005), Redistribution and Education Subsidies Are Siamese Twins, Journal of Public Economics 89, 2005 2035.

[3] Deaton, A. (1979), Optimally Uniform Taxes, Economics Letters 2, 357 - 361.

[4] Gauthier, S., and G. Laroque (2008), Separability and Public Finance, mimeo CREST-ENSAE.

[5] Guesnerie, R. (1995), A Contribution to the Pure Theory of Taxation, Econometric Society Monograph, Cambridge University Press, Cambridge, U.K.

[6] Hammond, P. (1979), Straightforward Individual Incentive Compatibility in Large Economies, Review of Economic Studies 46, 263 - 282.

[7] Hellwig, M.F. (2006/2008), Incentive Problems with Hidden Characteristics: A Unified Approach, Preprint 06-26, Max Planck Institute for Research on Collective Goods, Bonn, 2006, http://www.mpprdg.mpg.de/pdf_dat/2006_26online.pdf, revised 2008.

[8] Hellwig, M.F. (2008 a), A Maximum Principle for Control Problems with Monotonicity Constraints, Preprint 04/2008, Max Planck Institute for Research on Collective Goods, Bonn, Germany, http://www.coll.mpg.de/pdf_dat/2008_04online.pdf.

[9] Hellwig, M.F. (2008 b), Note on Deaton's (1979) Theorem on the Undesirability of Nonuniform Excise Taxaxation, Preprint ??/2008, Max Planck Institute for Research on Collective Goods, Bonn, http://www.coll.mpg.de/pdf_dat/2008_45online.pdf.

[10] Kaplow, L. (2006), On the Undesirability of Commodity Taxation Even When Income Taxation Is Not Optimal, Journal of Public Economics 90, $1235-1250$.

[11] Laroque, G. (2005), Indirect Taxation is Superfluous under Separability and Taste Homogeneity: A Simple Proof, Economics Letters 87, 141 144.

[12] Mirrlees, J.M. (1976), Optimal Tax Theoy: A Synthesis, Journal of Public Economics 6, 327 - 358. 